\title{
Review: radiotherapy reduces death from and recurrence of breast cancer but increases death from other causes
}

Early Breast Cancer Trialists' Collaborative Group. Favourable and unfavourable effects on long-term survival of
radiotherapy for early breast cancer: an overview of the randomised trials. Lancet 2000 May 20;355:1 757-70.

QUESTION: In women with early breast cancer, what are the long term (20 y) effects of radiotherapy on mortality from breast cancer and on mortality from causes other than breast cancer?

\section{Data sources}

Studies were identified by searching published lists of randomised trials and Medline; handsearching selected journals, meeting abstracts, and references of published trials and reviews; and by contacting experts repeatedly over a 15 year period.

\section{Study selection}

Studies were selected if they were randomised controlled trials begun before 1990 that compared radiotherapy plus other treatments with the same treatments without radiotherapy.

\section{Data extraction}

Information was requested for each woman on age; menopausal status; type of surgery and nodal status; and dates of randomisation, allocated treatment, first subsequent local recurrence, and death.

\section{Main results}

Data from 19582 women were assembled from 40 trials and analysed by intention to treat. Radiotherapy reduced local recurrence by about two thirds in all trials ( $10 \%$ v $30 \%$ by $20 \mathrm{y}, \mathrm{p}<0.001$, with most of this benefit in the first few years) and reduced 20 year breast cancer mortality in the absence of other causes of death (53.4\% $v 48.6 \%, \mathrm{p}<0.001$, with most of this benefit in the first decade) but increased mortality from other causes (20 y survival in the absence of deaths from breast cancer $69.5 \% v 73.8 \%, \mathrm{p}<0.001$, with most of the hazard after the first decade). The observed overall survival difference of $1.2 \%$ was not clinically or statistically significant $(\mathrm{p}=0.06)$

\section{Conclusions}

In women with early breast cancer (stages 1 and 2) and a substantial risk for local recurrence, radiotherapy reduces the risk for local recurrence by two thirds and reduces mortality from breast cancer by a few percentage points. Overall survival is improved only if the long term hazards are substantially smaller than the average in these trials.

\section{COMMENTARY}

This meta-analysis from the Early Breast Cancer Trialists Collaboration is important. Although radiation improved overall survival to a non-significant extent $(p=0.06)$, the interpretation is complex because of the inherent benefits and hazards. However, appropriate radiotherapy regimens may be able to retain most of the benefits while minimising the hazards.

Historical methods of radiation included high daily doses, overlapping fields, and larger irradiated volumes, all of which may increase mortality from causes other than breast cancer. The meta-analysis suggests that mortality from such causes may be lower with daily doses $<2.2$ Gy. However, such technical markers are analysed separately rather than in a multivariate analysis and are not statistically significant.

In the recent US National Institutes of Health consensus conference on adjuvant treatment of early breast cancer, postmastectomy radiotherapy was recommended only for patients with $\geqslant 4$ positive nodes. ${ }^{1}$ At issue now is whether the current practice of limiting radiation to breast and skin is sufficient where 0 to 3 nodes are positive. A randomised trial from the National Cancer Institute of Canada (MA-20) is addressing this question for 1 to 3 nodes.

Patients who survive breast cancer typically die from other common causes of death, especially cardiovascular diseases. An excess of vascular deaths was associated with nodal irradiation, including internal mammary nodes. Assigning cause of death remains problematic. Another published meta-analysis, confined to trials including systematic treatment, shows that overall mortality is significantly reduced $(\mathrm{p}=0.004$, odds ratio 0.83$)$ in trials using modern, and presumably safer, radiation techniques. ${ }^{2}$ However, mean follow up was only 10 years rather than the 20 years in this analysis, and further follow up is needed to clarify the relative survival benefit for radiotherapy.

Locoregional radiation administered with modern methods may improve overall survival in stages 1 and 2 breast cancer. The evidence suggests that many patients, particularly those who are younger and node positive, should consider nodal irradiation, even when systemic treatment is given.

Glenn W Jones, MD, MSc Hamilton Regional Cancer Centre Hamilton, Ontario, Canada

1 Adjuvant Therapy for Breast Cancer. NIH Consensus Statement. 2000 November 1-3;17:1-23. (http:// odp.od.nih.gov/consensus/cons/114/114 statement.htm)

2 Whelan TJ, Julian J, Wright J, et al. Does locoregional radiation therapy improve survival in breast cancer? A metaanalysis. J Clin Oncol 2000;18:1220-9. 